\title{
Design and experimental analysis of rice pneumatic seeder with adjustable seeding rate
}

\author{
He Xing ${ }^{1,2}$, Zaiman Wang ${ }^{1,2,3}$, Xiwen Luo ${ }^{1,2,3}$, Ying Zang ${ }^{1,2,3^{*}}$, Siyu He ${ }^{1,2}$, Peng $\mathrm{Xu}^{1,2}$, Shuncai Liu ${ }^{1,2}$ \\ (1. Key Laboratory of Key Technology on Agricultural Machine and Equipment, Ministry of Education, \\ South China Agricultural University, Guangzhou 510642, China; \\ 2. College of Engineering, South China Agricultural University, Guangzhou 510642, China; \\ 3. Guangdong Provincial Key Laboratory of Agricultural Artificial Intelligence (GDKL-AAI), Guangzhou 510642, China)
}

\begin{abstract}
To adapt to the diversity of hybrid rice and meet its requirements of precision seeding, a rice pneumatic seeder with adjustable seeding rates was designed in this study. The seeder employed an independent vortex pump to provide the air source for the seed metering device. According to the requirements of air flow in the seed metering device, a sub-pipeline and a general pipeline were designed. The performances of the two types of pipelines were verified by the air pressure uniformity tests. The results showed that the sub-pipeline can distribute the air pressure more evenly than the general pipeline. The coefficient of variation of the sub-pipeline pressure was less than 5\%. Wuyou 1179 hybrid rice was selected as the experimental object. The field seeding tests were carried out with different negative pressures and different numbers of groups of sucking holes as experimental factors. The results showed that the average qualified rate of seeding was $93.35 \%$ when only 2 groups of sucking holes were working under the seed sucking negative pressure of $2.0 \mathrm{kPa}$. The average qualified rate of seeding was $87.21 \%$ when 3 groups of sucking holes were working under the seed sucking negative pressure of $1.6 \mathrm{kPa}$. The average qualified rate of seeding was $83.73 \%$ when 4 groups of sucking holes were working under the seed sucking negative pressure of $1.6 \mathrm{kPa}$. The average qualified rate of seeding was $79.86 \%$ when 5 groups of sucking holes were working under the seed sucking negative pressure of $1.6 \mathrm{kPa}$. The main factors affecting the seeding effect were analyzed and the feasibility of a rice pneumatic seeder with adjustable seeding rates was verified by field tests. The research results can provide a basis for its practical production and application.
\end{abstract}

Keywords: agricultural machinery, adjustable seeding rate, seeder, pneumatic, rice, pipeline DOI: $10.25165 /$ j.ijabe.20211404.5658

Citation: Xing H, Wang Z M, Luo X W, Zang Y, He S Y, Xu P, et al. Design and experimental analysis of rice pneumatic seeder with adjustable seeding rate. Int J Agric \& Biol Eng, 2021; 14(4): 113-122.

\section{Introduction}

Rice is one of the three major food crops in China and the main economic food crop in the world. Rice is the staple food for more than half of the global population. At present, with the improvement in agricultural machinery and agronomic technology, mechanized planting and high-yielding crops are constantly updated, thus reducing the output of the labor force and achieving high returns. However, due to the characteristics of rice planting, it is difficult to mechanize it and, compared with other crops, the degree of mechanized planting of rice is low. It is the main reason that affects the entire mechanization of rice production ${ }^{[1-5]}$.

The technology of rice direct seeding consists of directly sowing rice seeds in the field. It eliminates the process of raising

\section{Received date: 2020-01-07 Accepted date: 2021-02-22}

Biographies: He Xing, Post-doctoral, research interest: agricultural mechanization and automation, Email: 675974347@qq.com; Zaiman Wang, Engineer, research interest: agricultural mechanization and automation, Email: wangzaiman@scau.edu.cn; Xiwen Luo, Professor, research interest: agricultural mechanization and automation, Email: xwluo@scau.edu.cn; Siyu He, MS candidate, research interest: agricultural electrification and automation, Email: 1141903213@qq.com. Peng Xu, MS candidate, research interest: agricultural electrification and automation, Email: 1731205919@qq.com; Shuncai Liu, MS candidate, research interest: agricultural electrification and automation, Email: 1572339289@qq.com.

*Corresponding author: Ying Zang, PhD, Professor, research interest: agricultural mechanization and automation. College of Engineering, South China Agricultural University, Guangzhou 510642, China. Tel: +86-20-38676975, Email: yingzang@scau.edu.cn. rice seedlings, reduces the input of the prophase cost, and increases the benefit. However, owing to the diversity of rice, different varieties need to adopt different sowing amounts, and the sowing amount needs to be adjusted when using direct seeding technology. Compared with conventional rice, hybrid rice has the characteristics of strong tillering ability and high yield. Precision seeding is needed with direct seeding technology. When different varieties of hybrid rice are sowed, the sowing amount is slightly different, which increases the difficulty of direct seeding of hybrid rice $^{[6,7]}$.

To adapt to the changes in crop sowing amounts caused by different varieties, scholars have conducted in-depth research on sowing technology. Zhou et al. ${ }^{[8]}$ designed an inside-filling adjustable precision seed-metering device with combined holes for cotton, which realized stepless adjustment of the seeding rate. The sowing precision of cotton seeds was tested, and the seeding precision met the requirements of cotton sowing. The seed metering device was added with a seed clearing device, which improved the seeding precision and provided a reference for optimization of the structure of the cotton seed metering device. Zhang et al. ${ }^{[9]}$ designed a combined hole-type metering device to meet the different seeding amount requirements of conventional rice and hybrid rice. The seed metering device adjusted the seeding amount by changing the hole type. Conventional rice and hybrid rice were selected as experimental objects. The results showed that the seed metering device could meet the application requirements of many types of rice, and they provided a reference basis for adjusting the seeding rate of the seed metering device. 
However, the seed metering device could only realize the change of two types of sowing amount and the seeding precision was not high; thus, it could not meet the requirements of small amount and precision in hybrid rice. Tian et al. ${ }^{[10]}$ designed a helical grooved rice seed metering device. The seeding rate was adjusted by changing the length of the spiral groove. The optimum working parameters were selected through experiments. It could meet the requirements of rice field sowing but the seeding precision of the seed metering device is not high. The main purpose was to sow conventional rice at a large seeding rate, and it was impossible to meet the requirements of precision seeding of hybrid rice. At the same time, the seed metering device used gravity to fill seeds, and it used rigid seed protection, which resulted in a high seed injury rate.

Compared with the mechanical seed metering device, the pneumatic seed metering device has the characteristics of high precision and low seed injury rate, and it has been widely used in the field of direct seeding. Ismet et al. ${ }^{[11]}$ studied the suction hole structure of the pneumatic metering device. The effects of the number of sucking holes and structure parameters on seed sucking were analyzed. The flow state of the airflow was also analyzed. Optimization experiments were carried out with soybean and maize, which provided a reference for the suction hole structure of the pneumatic seed metering device. Yasir $^{[12]}$ analyzed the seeding mechanism of the wheat pneumatic seed metering device, optimized its main working parts, carried out verification tests, and obtained the best structural and working parameters, thus providing a reference for the wheat pneumatic seed metering device. Karayel et al. ${ }^{[13-15]}$ carried out structural optimization experiments of a seed metering device with different crops. The relationship equation between the physical properties of different crops and the negative pressure was established. Yazgi et al. ${ }^{[16,17]}$ studied the vacuum, sucking hole diameter, number of sucking holes, and rotational speed of the sucking plate of the pneumatic seed metering device using a response surface methodology. The relationship equation between parameters and seeding uniformity was obtained. It provided a reference for uniform seeding of the pneumatic metering device. However, the research is still in the theoretical stage and has not been applied in practice. Zhang et al. ${ }^{[18]}$ designed a pneumatic seed metering device with multiple suction holes for rice. The seeding precision was improved by using a seed stirring device and a multi-suction hole structure. However, the seed metering device could not adjust the seeding rate and could not meet the requirements of the diversity of hybrid rice varieties. Dai et al. ${ }^{[18]}$ designed an air-blown seed metering device. A grooved wheel was used for seeding, and positive pressure was used for seed transportation. The seeding rate was changed by the rotational speed of the grooved wheel. However, the seeding uniformity of the seed metering device was poor. This seed metering device could only realize drilling and could not accurately and precisely sow.

The abovementioned studies had some shortcomings. The mechanical seed metering device with adjustable seeding rate could not achieve precision seeding, and the seeding rate was generally large. The mechanical seed metering device was mainly used with conventional rice. The pneumatic seed metering device had high seeding precision, but it could not achieve an adjustable seeding rate. These studies were conducted in the laboratory and have not been applied in the field. To solve the above problems, a rice pneumatic seeder with adjustable seeding rates was designed by using a rice pneumatic seed metering device with adjustable seeding rates. The working principle of the seeder was analyzed, and pressure pipes were designed to provide air power for the seed metering device. Field application tests of the seeder were carried out, and the working parameters were optimized, which provided a reference for the pneumatic precision seeding technology of rice.

\section{General structure and working principle of rice pneumatic seeder with adjustable seeding rate}

\subsection{General structure}

The general structure of the rice pneumatic seeder with adjustable seeding rates is shown in Figure 1. The pneumatic rice direct seeder mainly consists of a Yanmar (VP6D) rice transplanter, gasoline engine, vortex pump, negative pressure pipelines, positive pressure pipeline, ditching equipment, precision pneumatic rice seed-metering device with adjustable seeding rate, power output shaft, pontoon, and lifting frame.

The rice pneumatic seed metering device with adjustable seeding rates is shown in Figure 2. The seed metering device mainly comprises a negative pressure multi-flow path, which adjusts the seeding rate by changing the negative pressure flow paths. ${ }^{[20]}$

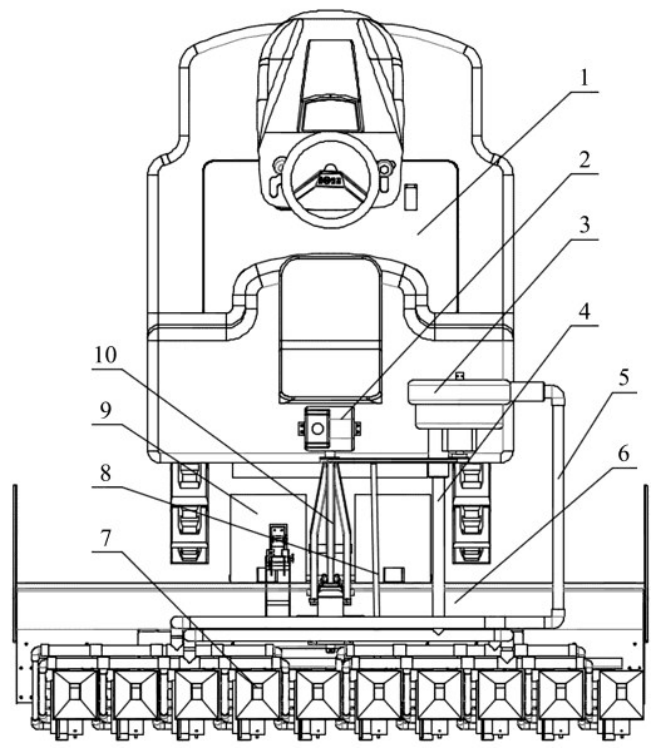

1. Yanmar (VP6D) rice transplanter 2. Gasoline engine 3. Vortex-pump 4. Negative pressure pipeline 5. Positive pressure pipeline 6. Ditching equipment 7. Precision pneumatic rice seed-metering device with adjustable seeding rate 8 . Power output shaft 9 . Pontoon 10 . Lifting frame.

Figure 1 Structure of rice pneumatic seeder with adjustable seeding rates

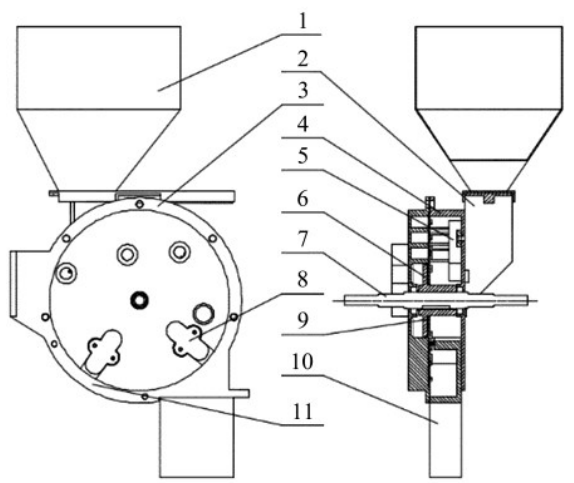

1. Seed box 2. Seed box connector 3. Gas chamber shell of multiple flow-paths 4. Seed chamber shell 5. Cleaning device 6. Flange 7. Shaft 8. Seed brush 9. Sucking plate 10. Seed tube 11. Seed unloading device

Figure 2 Structure diagram of rice pneumatic seed metering device with adjustable seeding rate 


\subsection{Working principle}

As shown in Figure 1, the seeding part of the rice pneumatic seeder with adjustable seeding rates was linked to the Yanmar (VP6D) rice transplanter by a three-point linkage system. The power was provided by the power output shaft of the transplanter and transmitted to the seed metering device through a chain transmission after deceleration through the reduction gearbox. The shafts of each one of the pneumatic rice seed-metering devices were connected with a coupling to ensure the synchronism of the rotation. A Honda gasoline engine was used to power the vortex pump. Positive and negative pressure pipelines were designed to connect the seed metering devices with the vortex pump, providing the gas source they needed. A piece of ditching equipment was used to create the sowing and water ditches. When the seed metering device was working, the seeds fell into the sowing ditch, and the water was held in the water ditch to ensure that the field surface was wet.

The diagram of the gas chamber shell with multiple flow paths is shown in Figure 3. Three independent negative pressure flow paths were set in the shell, and each negative pressure flow path was connected with a negative pressure interface. As shown in Figure 3, there were five groups of sucking holes on the sucking plate, which corresponded to three negative pressure flow paths. The No. 1 group of sucking holes corresponded to the No. 1 negative pressure flow path, the No. 2 and No. 3 groups of sucking holes corresponded to the No. 2 negative pressure flow path, and the No. 4 and No. 5 groups of sucking holes corresponded to the No. 3 negative pressure flow-path.

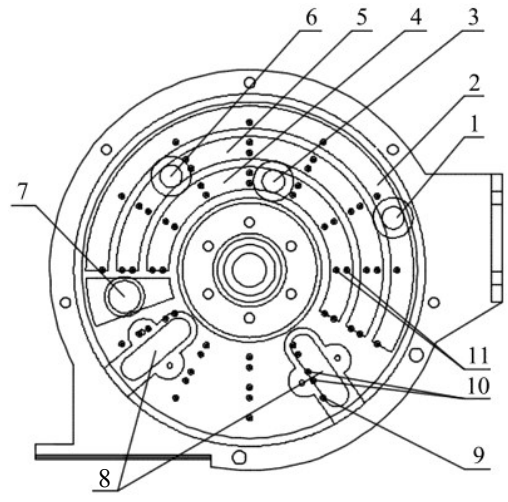

a. Gas chamber shell of multiple flow-paths

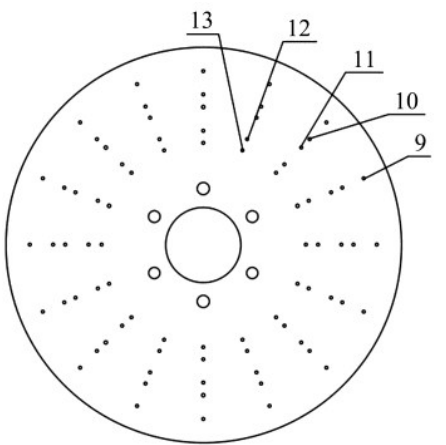

b. Sucking plate

1. No. 1 negative pressure interface 2. No. 1 flow-path 3 . No. 3 negative pressure interface 4. No. 3 flow-path 5. No. 2 flow-path 6. No. 2 negative pressure interface 7. Positive pressure chamber 8 . Seed brush 9. No. 1 group of sucking holes 10. No. 2 group of sucking holes 11. No. 3 group of sucking holes 12. No. 4 group of sucking holes 13. No. 5 group of sucking holes

Figure 3 Diagram of multiple flow-paths

When the seed metering device was working, the No. 1 negative pressure interface was opened, and the other two interfaces were closed. At this time, negative pressure was only generated in the No. 1 negative pressure flow path. Rice seeds were sucked on the No. 1 group of sucking holes. Because there was no negative pressure in the other two negative pressure flow paths, rice seeds would not be absorbed on the No. 2-5 groups of sucking holes. In this case, the seed metering device mainly absorbed single rice seeds. Similarly, when only the No. 2 negative pressure interface was opened, the negative pressure would be generated in the No. 2 negative pressure flow path. The rice seeds were absorbed on the No. 2 and No. 3 groups of sucking holes. At this time, two groups of sucking holes were working. In this case, the absorption seed precision of the seed metering device was mainly two seeds because it allowed multiple negative pressure flow paths to work at the same time. Therefore, a combined work was carried out between negative pressure flow paths. When the No.1 and No. 2 negative pressure interfaces were opened at the same time, the No. 1-3 groups of sucking holes would work at the same time. In this case, the absorption seed precision of the seed metering device was mainly three seeds. When the No. 2 and No. 3 negative pressure interfaces were opened at the same time, the No. 2-5 groups of sucking holes would work at the same time. In this case, the absorption seed precision of the seed metering device was mainly four seeds. When the No. 1-3 negative pressure interfaces were opened at the same time, the No. 1-5 groups of sucking holes would work at the same time. In this case, the absorption seed precision of the seed metering device was mainly five seeds.

\subsection{Vortex pump selection}

The RHG-7H3 vortex pump was selected, which has an output flow of $330 \mathrm{~m}^{3} / \mathrm{h}$, positive pressure of $39 \mathrm{kPa}$, and negative pressure of $-37 \mathrm{kPa}$. According to the preliminary tests, when the vortex pump worked at the maximum power, each seed metering device could reach $2.2 \mathrm{kPa}$ or more, which was larger than the optimum negative pressure of the seed metering device in the laboratory and met the negative pressure requirement of the seed metering device. Because there was no motor to power the vortex pump when the rice pneumatic seeder with adjustable seeding rate worked in the field, a gasoline engine was used to provide power output for the vortex pump. The output shaft of the vortex pump was connected with the output shaft of the gasoline engine by a belt. Because the output shaft speed of the gasoline engine was 1800-3600 r/min and the rated speed of the output shaft of the vortex pump was $2800 \mathrm{r} / \mathrm{min}$, the power transmission was carried out with a 1:1 transmission ratio. The installation drawings of the vortex pump and gasoline engine are displayed in Figure 4.

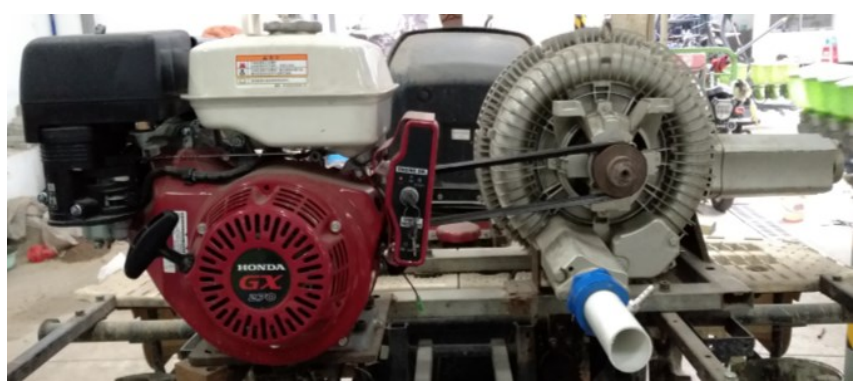

Figure 4 Vacuum air pump and gasoline

\subsection{Structural design and experimental study of pipeline}

Because the spacing between each seed metering device on the seeder was fixed, the pipe length between each seed metering device was also fixed. The air pressure loss was related to the 
length, bending, and diameter of the pipeline. According to fluid mechanics, there is a certain viscosity in air. When air flowed inside the pipeline, the boundary between the air and the pipeline did not slip, and the air velocity on the boundary was zero. The velocity distribution of air passing through the cross section of the pipeline is not uniform in a straight flow. There would be relative motion between two adjacent flow layers, and thus internal friction was produced. The increase in pipeline length would increase the internal friction of the flow, which would increase the energy loss of the fluid and cause pressure loss, that is, the pressure loss along the way. Because of the change in the boundary of the flow path, the velocity in the cross section will change dramatically. This will create a vortex. The relative motion between fluid particles will be strengthened during the reorganization of the flow velocity distribution, which leads to an increase in internal friction and energy loss. The rapid change in the velocity distribution in the cross section was caused by the local sharp change. The additional force caused by this is local resistance. The pressure loss caused by this is the local pressure loss ${ }^{[21]}$.

When the flow rate does not change, an increase in pipeline diameter can reduce the flow velocity in the pipeline. When the flow velocity decreases, the friction between the fluid and the boundary can be reduced and the loss along the flow path can be reduced. Therefore, the flow in a pipeline with a relatively large diameter is conducive to a reduction in pressure loss. To cooperate with the structure of the seed metering device, a large pipeline will not be used. When the pipeline diameter is excessively large, it will lead to a change of the diameter at the junction. This will increase the local pressure loss. At the same time, to coordinate the interface size of the vacuum pump, the pipeline size of the front section was designed to have the same size as that of the vacuum pump interface, that is, a $50 \mathrm{~mm}$ pipeline was selected to connect with the vacuum pump. Because the size of the negative pressure interface of the seed metering device was $25 \mathrm{~mm}$, a tee joint of 50 to $25 \mathrm{~mm}$ was used to connect the hose. The inner diameter of the hose was $25 \mathrm{~mm}$. Then, the hose was connected with the negative pressure interface of the seed metering device, which could reduce the local pressure loss and pressure loss along the way.

To distribute the air pressure evenly and reduce the loss of air pressure in the process of air transportation, two types of pipelines were designed, as shown in Figure 5. Figure 5a shows a sub-pipeline, which divided the main pipeline into two parts and redistributed the two branch pipelines to five branch pipelines. The sub-pipelines were connected to the negative pressure interface of the seed metering device. Figure $5 \mathrm{~b}$ depicts the general pipeline, which was directly divided into 10 parts and connected to the negative pressure interface of the seed metering device.

To study the uniformity and stability of the pressure distribution of the two types of pipelines, experiments were carried out under different negative pressures of the main pipeline. The pressure value of each sub-pipeline was measured by a horizontal pressure gauge. In addition, the horizontal pressure gauge was used to measure the pressure accurately. First, a threaded hole was made on the pipeline. Then, a threaded joint was installed in the threaded hole, and the waterproof tape was wrapped around the joint to prevent pressure leakage from the hole. A rubber hose was used to connect the joint with the horizontal pressure gauge, which measured the pressure value. The metering device was in a static state when the air pressure was measured.

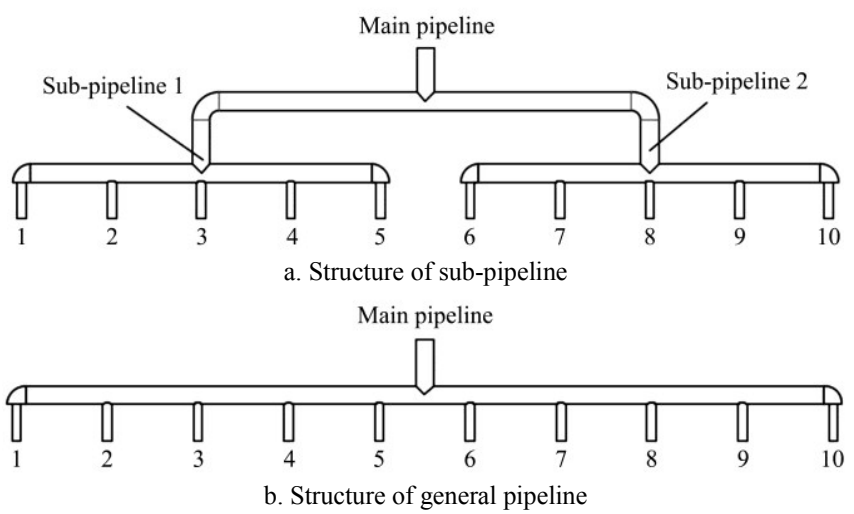

Note: The numbers (1-10) in the figure are serial numbers of seed metering devices of the seeder

Figure 5 Pipelines connected to the vacuum pump and the seeder

The test results of the sub-pipeline are presented in Table 1. The test results showed that the negative pressure of the main pipeline was $4.0 \mathrm{kPa}$, and the average pressure of the branch pipeline was $2.05 \mathrm{kPa}$. The coefficient of variation was $3.13 \%$. When the negative pressure of the main pipeline was $6.0 \mathrm{kPa}$, the average pressure of the branch pipeline was $2.38 \mathrm{kPa}$. The coefficient of variation was $3.15 \%$. When the negative pressure of the main pipeline was $8.0 \mathrm{kPa}$, the average pressure of the branch pipeline was $3.00 \mathrm{kPa}$. The coefficient of variation was $3.53 \%$. All the coefficients of variation were less than 5\%, which met the requirement of less than 5\%. According to the test results, the pressure of the No.3 and No.8 metering devices was higher than the pressure of other metering devices. The main reason was that the main pipeline was divided into two parts by the sub-pipeline, and the positions of the No.3 and No.8 seed metering devices were in the middle of the pipeline; thus, their air pressure was higher than that of other seed metering devices. The farther the seed metering device was from the middle seed metering device, the lower its pressure. The main reason was that the pressure loss along the way increased with the increase in pipeline length, and therefore the pressure of the two ends seed metering device was lower than the pressure of the seed metering device in the middle position.

Table 1 Test results of sub-pipeline (kPa)

\begin{tabular}{|c|c|c|c|c|c|c|c|c|c|c|c|c|}
\hline \multirow{2}{*}{$\begin{array}{l}\text { Main pipeline } \\
\qquad / \mathrm{kPa}\end{array}$} & \multicolumn{10}{|c|}{ Seed metering device number } & \multirow{2}{*}{ Average } & \multirow{2}{*}{$\mathrm{CV} / \%$} \\
\hline & 1 & 2 & 3 & 4 & 5 & 6 & 7 & 8 & 9 & 10 & & \\
\hline 4.00 & 1.98 & 2.05 & 2.13 & 2.02 & 1.96 & 2.02 & 2.08 & 2.15 & 2.10 & 2.00 & 2.05 & 3.13 \\
\hline 6.00 & 2.28 & 2.36 & 2.45 & 2.40 & 2.30 & 2.32 & 2.44 & 2.50 & 2.40 & 2.30 & 2.38 & 3.15 \\
\hline 8.00 & 2.85 & 2.98 & 3.10 & 3.04 & 2.87 & 2.90 & 3.06 & 3.14 & 3.10 & 2.92 & 3.00 & 3.53 \\
\hline
\end{tabular}

The test results of the general pipeline are listed in Table 2 . The test results show that when the negative pressure of the main pipeline was $4.0 \mathrm{kPa}$, the average pressure of the branch pipeline was $2.18 \mathrm{kPa}$. The coefficient of variation was $6.37 \%$. When the negative pressure of the main pipeline was $6.0 \mathrm{kPa}$, the average pressure of the branch pipeline was $2.57 \mathrm{kPa}$. The coefficient of variation was $6.52 \%$. When the negative pressure of the main pipeline was $8.0 \mathrm{kPa}$, the average pressure of the branch pipeline 
was $3.34 \mathrm{kPa}$. The coefficient of variation was $8.10 \%$. The coefficients of variation were all more than 5\%. According to the test results, the pressures of the No. 5 and No. 6 seed metering devices in the middle position were relatively high. The farther the seed metering device was from that at the middle, the lower its pressure. The air pressures of the seed metering devices at both ends were the lowest. The main reason was that the position of the main pipe was between the No.5 and No.6 seed metering devices, and thus the pressure of the No.5 and No.6 seed metering devices was higher. Because of the pressure loss along the way, the pressure at both ends decreased with the increase in pipeline length.

Compared with the test results of the sub-pipeline, the average pressure of the general pipeline was higher than that of the sub-pipeline, but its coefficient of variation was also higher. The main reason was that the main pipeline of the sub-pipeline was divided into two branch pipelines, and then the two branch pipelines were divided into 10 branch pipelines for the 10 seed metering devices. This distribution mode increased the pressure distribution steps, that is, the pressure loss along the pipelines was increased, which led to the increase in air pressure loss. The average air pressure of the sub-pipeline was less than the average air pressure of the general pipeline. However, the main pipeline of the sub-pipeline was divided into two branch pipelines, and then the two branch pipelines were divided into 10 seed metering devices. There were only five seed metering devices in each section. In this way, the number of seed metering devices was reduced, and the pipeline length was also reduced; hence, the five seed metering devices could distribute the air pressure more uniformly. The air pressure difference between the seed metering devices was reduced, and the coefficient of variation of air pressure between the seed metering devices was reduced. In summary, the sub-pipeline was selected because it could distribute the air pressure more evenly.

Table 2 Test results of pressures of the branch pipelines with different main pipeline pressures (kPa)

\begin{tabular}{|c|c|c|c|c|c|c|c|c|c|c|c|c|}
\hline \multirow{2}{*}{$\begin{array}{l}\text { Negative pressure of } \\
\text { the main pipeline } \\
/ \mathrm{kPa}\end{array}$} & \multicolumn{10}{|c|}{ Seed metering device number } & \multirow{2}{*}{ Average } & \multirow{2}{*}{$\mathrm{CV} / \%$} \\
\hline & 1 & 2 & 3 & 4 & 5 & 6 & 7 & 8 & 9 & 10 & & \\
\hline 4.00 & 2.03 & 2.10 & 2.21 & 2.23 & 2.35 & 2.42 & 2.25 & 2.16 & 2.05 & 2.00 & 2.18 & 6.37 \\
\hline 6.00 & 2.34 & 2.36 & 2.55 & 2.62 & 2.75 & 2.80 & 2.77 & 2.58 & 2.50 & 2.42 & 2.57 & 6.52 \\
\hline 8.00 & 2.95 & 3.22 & 3.34 & 3.52 & 3.65 & 3.70 & 3.52 & 3.40 & 3.11 & 2.96 & 3.34 & 8.10 \\
\hline
\end{tabular}

\section{Materials and methods}

\subsection{Test materials}

To test and analyze the field seeding precision of the pneumatic direct seeder with adjustable seeding rate, the super hybrid rice Wuyou 1179 was selected as the test material.

In this study, the size of the super hybrid rice Wuyou 1179 was measured by testing 100 rice seeds and the test was repeated three times. The average size of the seed was $8.94 \mathrm{~mm} \times 2.30 \mathrm{~mm} \times$ $1.94 \mathrm{~mm}$ (length $\times$ width $\times$ thickness). The weight of 1000 grains was $23.09 \mathrm{~g}$. The seeds were washed with water to remove impurities and blighted grain. The dry seeds were soaked in clear water for $24 \mathrm{~h}$. Then, they were moistened to bud, filtered out, and dried. The bud length was less than $3 \mathrm{~mm}$ by controlling the time of germination. Thus, the bud was short and hard, and it would not suffer damage in the process of seed sucking, nor would it cause blockage to the seed sucking hole. The average moisture content was $22.5 \%$ (wet basis) before the test.

The test was carried out in the Cencun Experimental Farm of South China Agricultural University. Before the test, the field had been soaked for $2 \mathrm{~d}$, and a rotary cultivator was used to plow the field with water twice until there was mud in the field. A laser land levelling machine was used for levelling the paddy field. The mud was precipitated for $2 \mathrm{~d}$ before seeding. The field was ditched well after precipitation of the paddy field to facilitate recording data after seeding. The test date was 9th August 2018, and the local temperature was $35^{\circ} \mathrm{C}$

\subsection{Test methods}

To observe the test results clearly, the hill spacing of the test prototype was fixed at $180 \mathrm{~mm}$. According to the literature ${ }^{[19]}$, when the negative pressure is $1.6 \mathrm{kPa}$, the optimal seeding precision can be reached. However, due to the complexity of the field conditions and to find the best sucking negative pressure, $1.2 \mathrm{kPa}, 1.6 \mathrm{kPa}$, and $2.0 \mathrm{kPa}$ were selected as the test negative pressures. The tests were carried out under different negative pressures and different numbers of sucking holes. The test factors are presented in Table 3. The seeding amount was small when using only one group of sucking holes, and thus sowing with only one group of sucking holes was barely used in the field. Therefore, 2-5 groups of sucking holes were selected as test levels. The application of the seeder in the field required a high working efficiency, and a high field seeding speed was needed. In this study, the seed speed was fixed to $1.0 \mathrm{~m} / \mathrm{s}$. It was difficult to keep the speed constant when the seeder was working in the complex field, but it could basically be stabilized at approximately $1 \mathrm{~m} / \mathrm{s}$. It was known from the literature ${ }^{[7]}$ that when the hill spacing was $180 \mathrm{~mm}$, the number of sucking hole groups was 12 , the forward speed of the seeder was $1 \mathrm{~m} / \mathrm{s}$, and the corresponding rotational speed of the seed metering device was $27.78 \mathrm{r} / \mathrm{min}$.

Table 3 Level table of test factors

\begin{tabular}{ccc}
\hline & \multicolumn{2}{c}{ Factor } \\
\cline { 2 - 3 } Level & A. Negative pressure/kPa & B. Sucking holes number \\
\hline 1 & 1.2 & 2 \\
2 & 1.6 & 3 \\
3 & 2.0 & 4 \\
4 & -- & 5 \\
\hline
\end{tabular}

Following the regulations of the Chinese National Standard $(\mathrm{GB} / \mathrm{T} \text { 6973-2005) })^{[22]}$, the seed amount for every hill was continuously recorded. The seed metering devices were numbered from 1 to 10 from left to right, and the seeding precision of each seed metering device was recorded. To each seed metering device were assigned 100 hills, and this was repeated three times (300 hills per seed metering device). The statistical data were analyzed using Matlab software.

\subsection{Field test results and analysis}

The field test results for two groups of holes are shown in Figure 6. Figure 6a shows that the probability of missed seed decreased with the increase in seed-sucking negative pressure. When the negative pressure increased from 1.2 to $1.6 \mathrm{kPa}$, the probability of missed seed decreased by $8.64 \%$. When the 
negative pressure increased from 1.6 to $2.0 \mathrm{kPa}$, the probability of missed seed decreased by $5.62 \%$. The degree of reduction from 1.6 to $2.0 \mathrm{kPa}$ was smaller than that from 1.2 to $1.6 \mathrm{kPa}$. The main reason was that the area of a sucking hole was limited. The seed sucking capacity of sucking holes was limited, and it could not be infinitely improved. When the negative pressure of seed sucking reached a critical value, the seed sucking amount would no longer increase. This was the saturated state of seed sucking. Figure 6a shows that there are differences in the probabilities of missed seeds of each seed metering device. The probability of missed seed was wavy. The main reason was the uneven distribution of air pressure. According to the pipeline structure, the No. 3 and No. 8 seed metering devices were in the middle position. Their air pressures were higher than those at the two ends, and hence their seed sucking capacity was also greater than that of other seed metering devices, and the probability of missed seed was lower. Figure $6 \mathrm{~b}$ shows that when the negative pressure of seed sucking was $2.0 \mathrm{kPa}$, the qualified rate was higher than that under the other conditions. The optimum negative pressure of seed sucking was $2.0 \mathrm{kPa}$. When the negative pressure was $2.0 \mathrm{kPa}$, the fluctuation of the qualified rate was also less than that

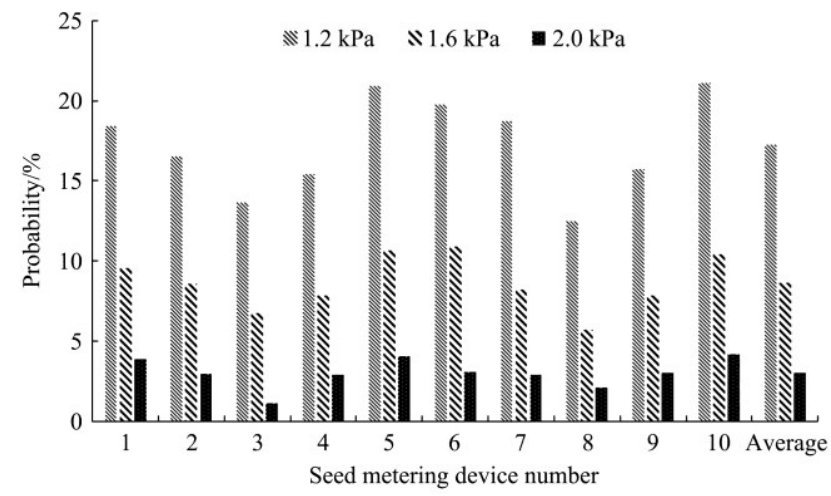

a. Missed seeds per hill

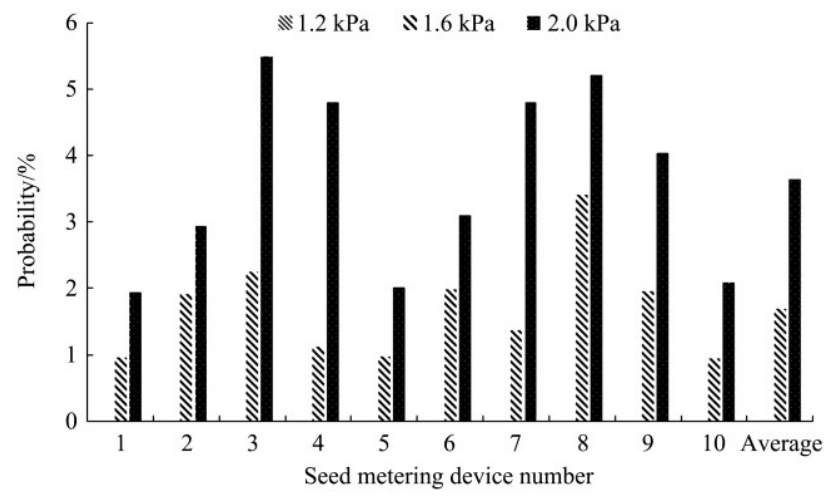

c. Re-adsorption rates (probability of $\geq 4$ seeds per hill) under other negative pressure conditions. The stability was higher when the negative pressure was $2.0 \mathrm{kPa}$. When the negative pressure was $1.2 \mathrm{kPa}$, there was a great difference in the qualified rate of seeding among the seed metering devices. The main reason for this phenomenon was that the gap between $1.2 \mathrm{kPa}$ and the optimum negative pressure was large. The pipeline tests showed that the negative pressures of the No. 3 and No. 8 seed metering devices were closer to the optimum suction negative pressure than those of other seed metering devices. According to Figure $6 \mathrm{c}$, the re-absorption rate of each seed metering device was $0 \%$ at $1.2 \mathrm{kPa}$, which indicated that the sucking state was far from the saturation state under this pressure. Therefore, under this condition, a slight increase in negative pressure could lead to a greater increase in the qualified rate of seeding. Figure $6 \mathrm{~d}$ shows that the average seeding amount under a negative pressure of $1.2 \mathrm{kPa}$ was much smaller than that under other conditions, which indicates that there was a big gap between $1.2 \mathrm{kPa}$ and the optimum negative pressure of seed sucking. When the negative pressure was $2.0 \mathrm{kPa}$, the average seeding amount was approximately two seeds. Therefore, the optimum seed sucking negative pressure for two groups of sucking holes seeding was $2.0 \mathrm{kPa}$.

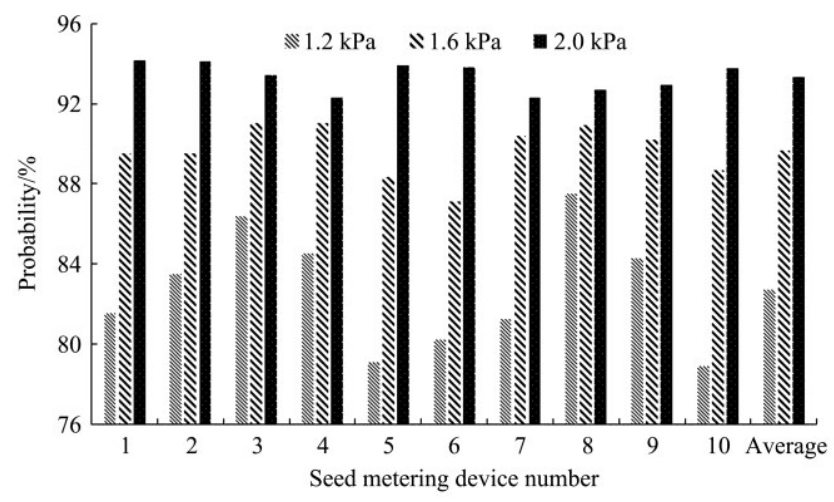

b. Qualified rates (1-3 sucked seeds per hill)

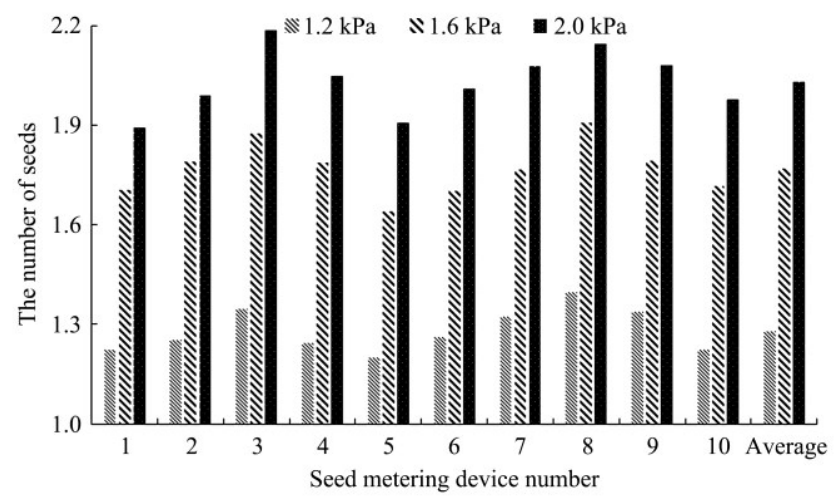

d. Average number of seeds

Figure 6 Test results of two groups of sucking holes

The test results for three groups of holes are shown in Figure 7. As depicted in Figure 7a, when the seed sucking negative pressure was $1.2 \mathrm{kPa}$, the average probability of $\leq 1$ seed per hill was $23.90 \%$. It was much higher than the $6.08 \%$ of $1.6 \mathrm{kPa}$ and $3.00 \%$ of $2.0 \mathrm{kPa}$. The fluctuation of the probability of $\leq 1$ seed per hill between the seed metering devices was higher than that at 1.6 and $2.0 \mathrm{kPa}$. The main reason for this phenomenon was the same as that for the probability of missed seed of two groups of holes. The main reason was that a negative pressure of $1.2 \mathrm{kPa}$ was far from reaching the saturated seed sucking negative pressure, and thus the average probability of $\leq 1$ seed per hill was much lower than the average probability of $\leq 1$ seed per hill under other conditions. Figure $7 \mathrm{~b}$ shows that when the sucking negative pressure is $1.6 \mathrm{kPa}$, the qualified rate of seeding was higher than that under other conditions, and the average qualified rate of seeding is $87.21 \%$. However, this average qualified rate of seeding was lower than that obtained in the laboratory tests. The main reasons for this phenomenon were as follows: 1) the sowing environment in the field was complex, and the seeder was affected by many external environmental factors, such as mechanical vibration. 2) The distribution of air pressure in the pipeline was uneven. Because of the use of a sub-pipeline, there were differences in the negative pressure of seed sucking of each seed metering device. When some seed metering devices reached the 
optimum air pressure, the other seed metering devices could not reach the optimum negative pressure of seed sucking, which resulted in a decrease in the average qualified rate of seeding. 3) The vortex pump obtained power from a gasoline engine, but the power stability of this engine was poor, and hence the negative pressure fluctuation was large. The vibration generated by the gasoline engine would also affect the vortex pump when sowing in the field, and the poor stability of negative pressure would affect the seeding precision. As shown in Figure 7c, when the negative pressure was $2.0 \mathrm{kPa}$, the average seed re-absorption rate was
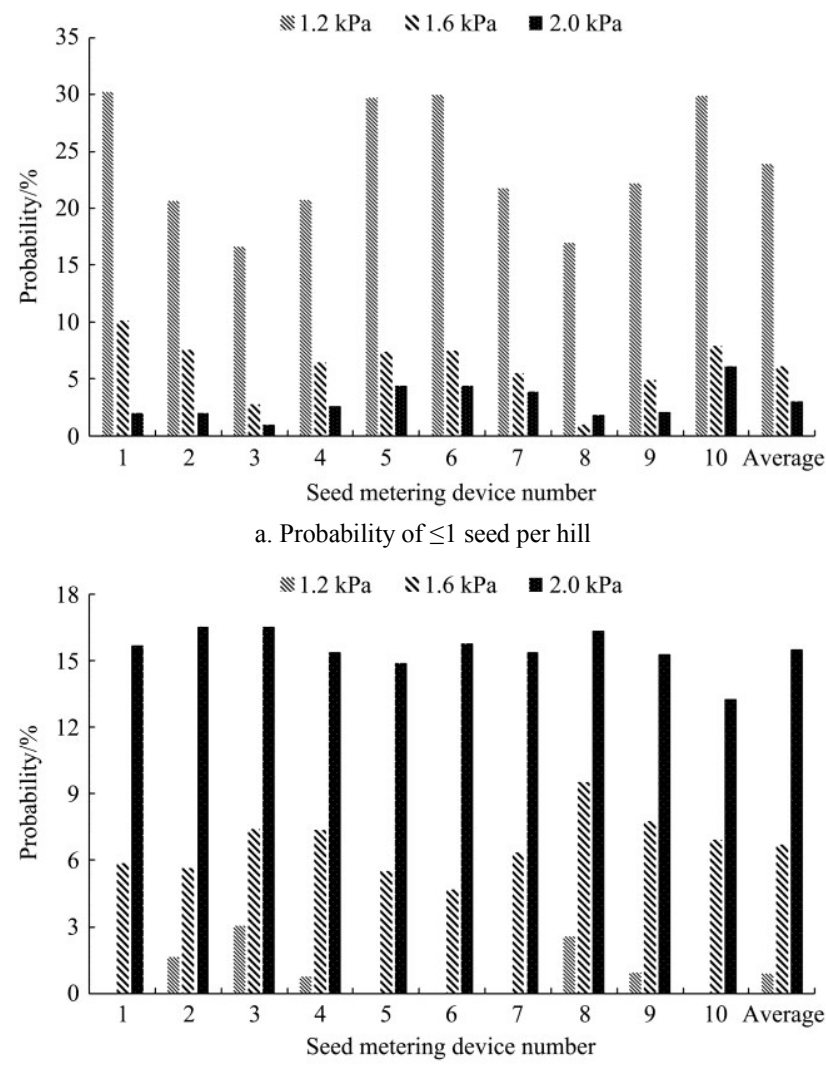

c. Re-adsorption rate (probability of $\geq 5$ seeds per hill)
$15.51 \%$, which was higher than that under other conditions. The results showed that when three groups of sucking holes were seeding, the sucking seed saturation would lead to a higher re-absorption rate and a lower qualified rate of seeding. The results revealed that there was a large differential pressure from the pressure in the saturation state of sucking seed to $1.6 \mathrm{kPa}$. As shown in Figure $7 \mathrm{~d}$, when the sucking negative pressure was $1.6 \mathrm{kPa}$, the average number of seeds per hole was 2.90 , which was close to three seeds per hill, which met the requirements for three sucking holes in the field.

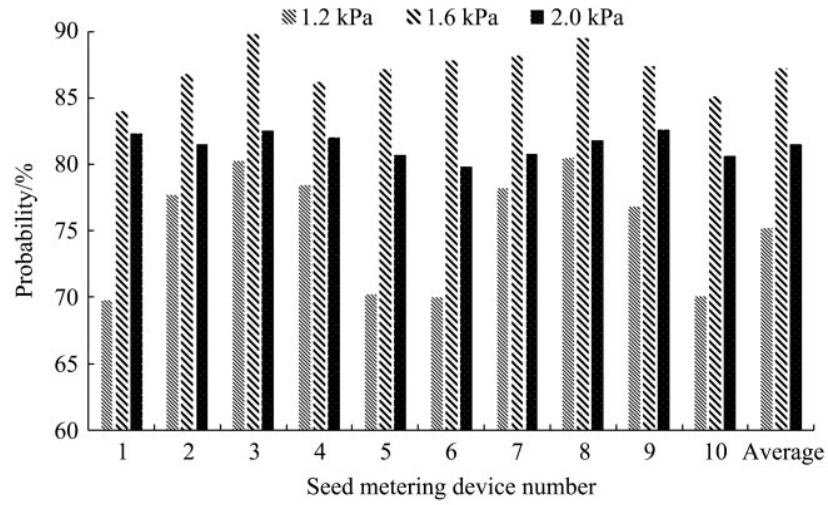

b. Qualified rate (2-4 sucked seeds per hill)

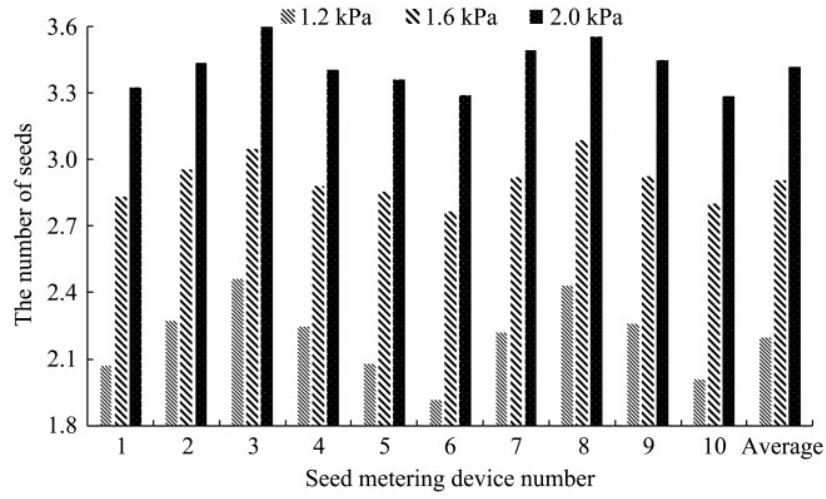

d. Average number of seeds

Figure 7 Test results of three groups of sucking holes

The test results for four groups of sucking holes are exhibited in Figure 8. According to Figure 8a, when the negative pressure of seed sucking was $1.2 \mathrm{kPa}$, the average probability of $\leq 2$ seeds per hill was $33.56 \%$. This value was much larger than the average probabilities of $\leq 2$ seeds per hill under $1.6 \mathrm{kPa}$ and $2.0 \mathrm{kPa}$. This further proved that a larger negative sucking pressure was needed to achieve the seed sucking saturation state under the condition of multiple suction holes. Figure $8 \mathrm{~b}$ shows that when the sucking negative pressure was $1.6 \mathrm{kPa}$, the seeding qualified rate was higher, the fluctuation of qualified rate between the seed metering devices was smaller, and the stability was higher, which met the requirements of field seeding. From Figures $8 \mathrm{c}$ and $8 \mathrm{~d}$, it can be observed that the distribution of pipeline pressure has an effect on the seeding precision, which was similar to the test results for three groups of sucking holes. Because of the uneven distribution of air pressure in the pipeline, there was a fluctuation in seed sucking which affected the test results. According to Figure 8, the optimum average qualified rate for four groups of sucking holes was $83.73 \%$, which met the requirements of field seeding.

The test results for 5 groups of sucking holes are exhibited in Figure 9. Figure $9 \mathrm{a}$ reveals that the average probability of $\leq 3$ seeds per hill is $29.43 \%$ when the negative pressure of seed sucking was $1.2 \mathrm{kPa}$. The average probability of $\leq 3$ seeds per hill of the 10 seed metering devices was $9.61 \%$ when the negative pressure of seed sucking was $1.6 \mathrm{kPa}$. The average probability of $\leq 3$ seeds per hill of 10 seed metering devices was $7.15 \%$ when the negative pressure of seed sucking was $2.0 \mathrm{kPa}$. The negative pressure of seed sucking increased from 1.6 to $2.0 \mathrm{kPa}$, and the average probability of $\leq 3$ seeds per hill increased by only $2.46 \%$. This indicated that the average probability of $\leq 3$ seeds per hill increased slowly in this negative pressure interval, and it was close to the saturation of seed sucking. Figure $9 \mathrm{~b}$ shows that when the negative pressure of seed sucking is $1.6 \mathrm{kPa}$, the optimum negative pressure of seed sucking is reached, and the average qualified rate of seed seeding is $79.86 \%$. Figure 9c shows that the average probability of re-absorption is $10.53 \%$ when the negative pressure of seed sucking is $1.6 \mathrm{kPa}$. The main reason for this phenomenon was that when five groups of sucking holes were working at the same time, each sucking hole could reabsorb seeds. This led to an increase in the re-absorption rate under the condition of multiple sucking holes. The re-absorption rate of sucking holes would also be increased with the increase of seeding rates. Therefore, when five groups of sucking holes were working at the same time, the 
re-absorption rate was higher. Figure $9 \mathrm{~d}$ shows that when the sucking negative pressure is $1.6 \mathrm{kPa}$, the average seeding rate is 4.95 seeds per hill, which is close to five seeds per hill. The

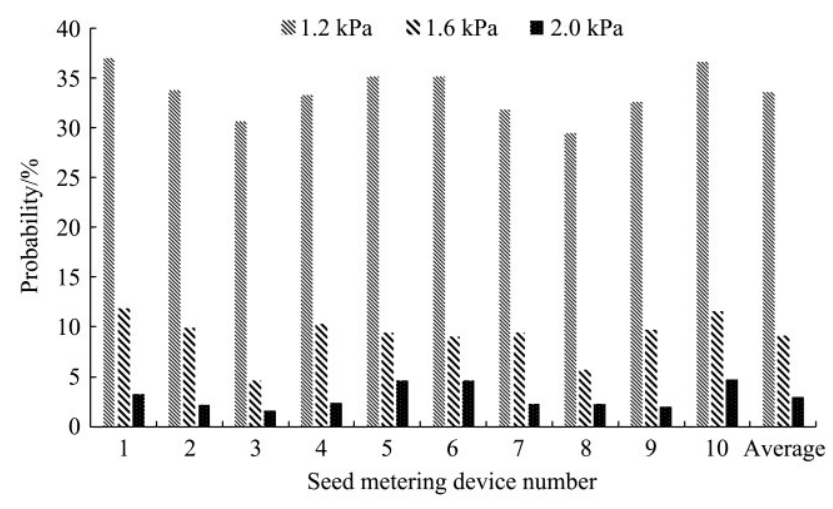

a. Probability of $\leq 2$ seeds per hill

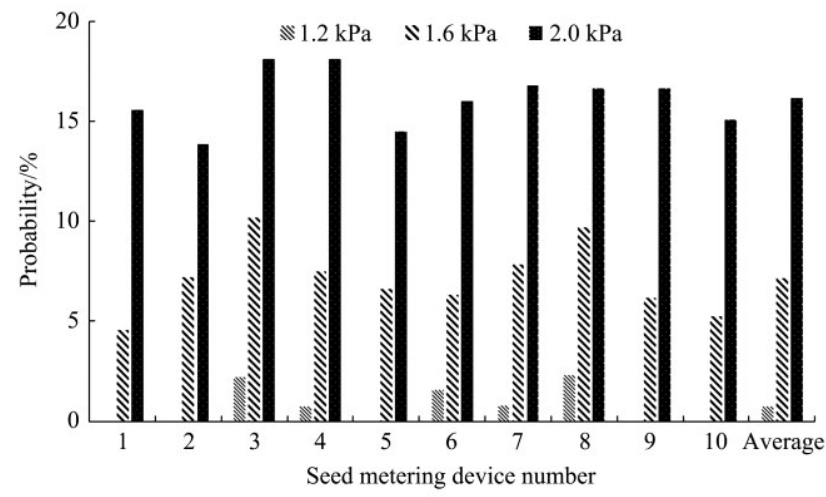

c. Re-adsorption rate (probability of $\geq 6$ seeds per hill) results showed that the average seeding rate reached the requirement for five groups of holes and met the requirements of field seeding.

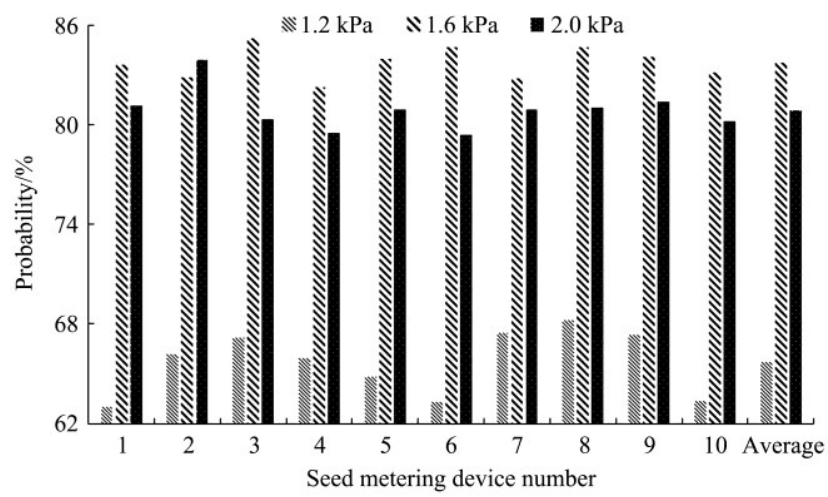

b. Qualified rate (3-5 sucked seeds per hill\%)

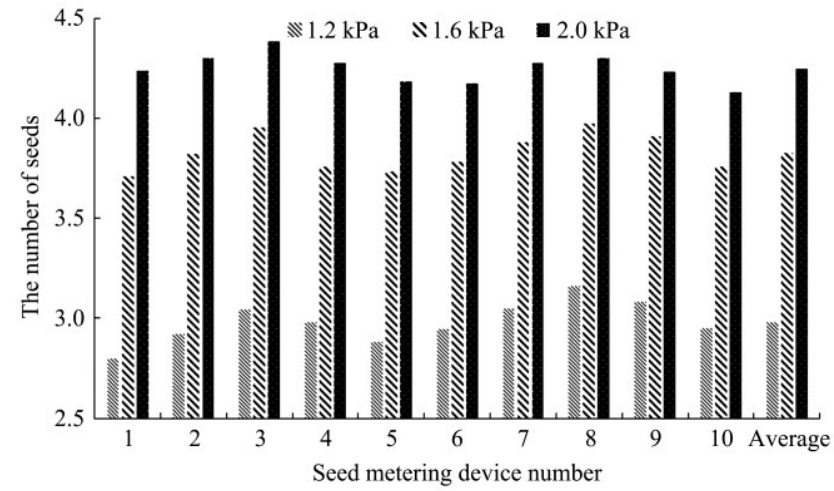

d. Average number of seeds

Figure 8 Test results of four groups of sucking holes

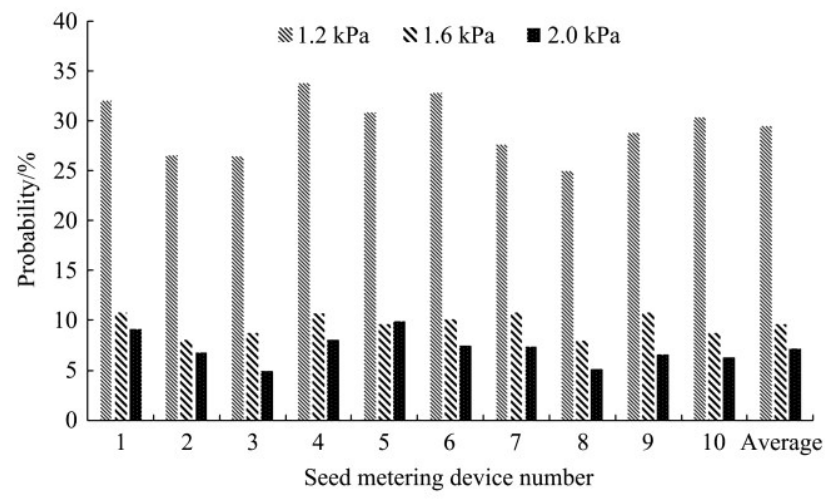

a. Probability of $\leq 3$ seeds per hill

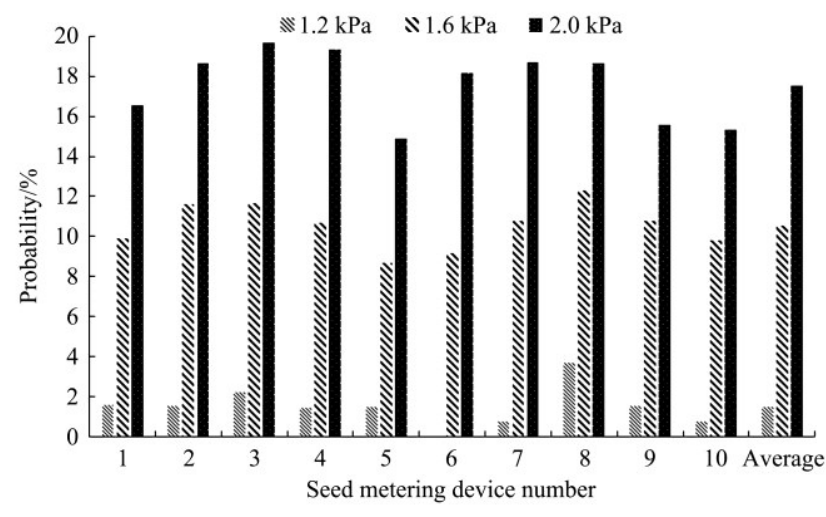

c. Re-adsorption rate(probability of $\geq 7$ seeds per hill)

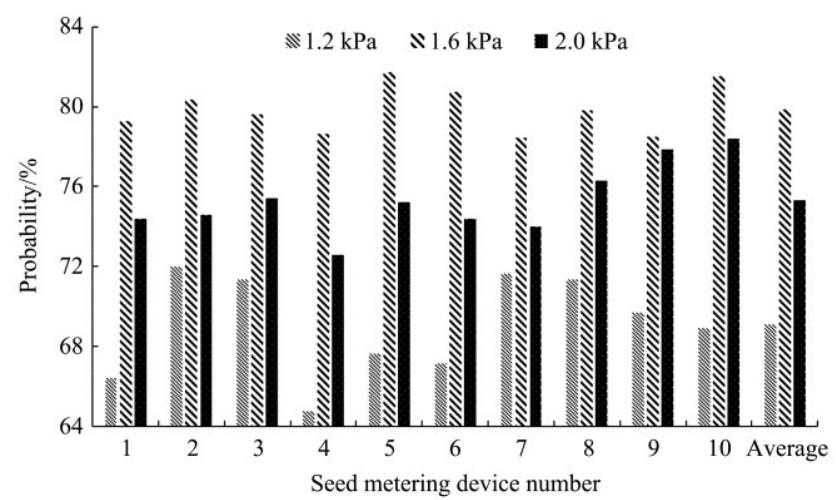

b. Qualified rate (4-6 sucked seeds per hill)

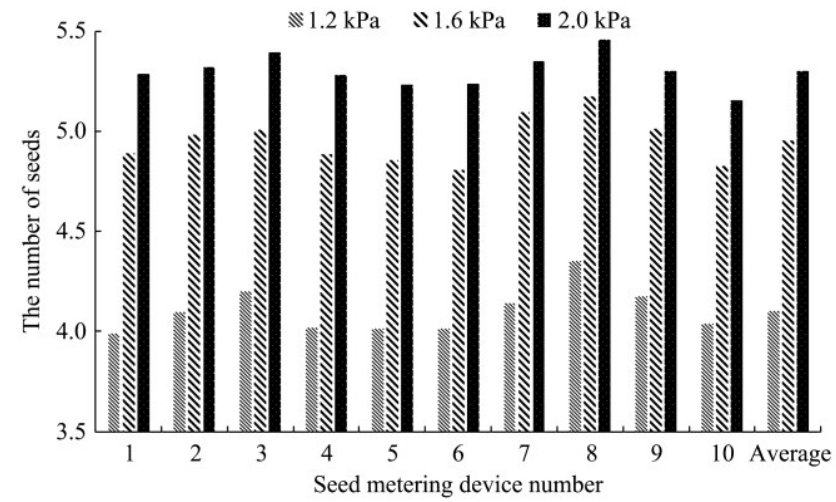

d. Average number of seeds

Figure 9 Test results of five groups of sucking holes

The coefficient of variation among the seed metering devices is presented in Table 4. When two groups of sucking holes were working, the average re-absorption rate was $0 \%$ under $1.2 \mathrm{kPa}$, which resulted in no coefficient of variation. It was found that the 
coefficients of variation of the re-absorption rate were over $100 \%$ when there were three or four groups of sucking holes were working. The main reason was that the low negative pressure of seed sucking would cause that the re-absorption rate of a seed metering device was $0 \%$. In this case, the slight change in the re-absorption rate would cause a relatively large change in the coefficients of variation. At the same time, it was found that the greater the negative pressure of seed sucking was, the smaller the coefficient of variation and the more stable the fluctuation of the reabsorption rate. This showed that the closer to the saturation state of seed sucking, the more stable the amount of seed sucking was, and the less likely it was to change. Comparing the probabilities of missed seed, it was found that the greater the negative pressure of seed sucking was, the greater the coefficient of variation, which indicated that the increase in negative pressure of seed sucking could not only reduce the probability of missed seeds, but also increase the fluctuation of the probability of missed seed. As can be observed in Table 4, the highest coefficient of variation of the qualified rate of seeding was $5.53 \%$, and the highest coefficient of variation of the average sowing amount was $7.32 \%$ under a negative suction pressure of $1.2 \mathrm{kPa}$. The results revealed that increasing the seed suction negative pressure was helpful to improve the stability of the qualified rate and average seeding amount. The coefficients of variation were less than $5 \%$ when the seed sucking negative pressure was 1.6 or $2.0 \mathrm{kPa}$, and the fluctuation of the coefficients of variation was small, which met the requirement of seeding uniformity for the direct seeder. Combining this with the results in Figures 6-9, the optimum seed sucking negative pressure with two sucking holes was $2.0 \mathrm{kPa}$, and that with 3-5 sucking holes was $1.6 \mathrm{kPa}$.

Table 4 Coefficient of variation (CV) among the seed metering devices (\%)

\begin{tabular}{|c|c|c|c|c|c|c|c|c|}
\hline \multirow{2}{*}{ Test factors } & \multicolumn{4}{|c|}{$\mathrm{CV}$ of 2 Holes } & \multicolumn{4}{|c|}{$\mathrm{CV}$ of 3 Holes } \\
\hline & $\begin{array}{c}\text { Missed seed } \\
\text { per hill }\end{array}$ & $\begin{array}{c}\text { 1-3 sucked } \\
\text { seed per hill }\end{array}$ & $\begin{array}{c}\geq 4 \text { sucked } \\
\text { seed per hill }\end{array}$ & Average & $\begin{array}{c}\leq 1 \text { sucked } \\
\text { seed per hill }\end{array}$ & $\begin{array}{c}1-3 \text { sucked } \\
\text { seed per hill }\end{array}$ & $\begin{array}{c}\geq 5 \text { sucked } \\
\text { seed per hill }\end{array}$ & Average \\
\hline $1.2 \mathrm{kPa}$ & 15.62 & 3.26 & -- & 4.60 & 20.97 & 5.53 & 116.45 & 7.32 \\
\hline $1.6 \mathrm{kPa}$ & 18.07 & 1.31 & 41.98 & 4.18 & 39.75 & 1.86 & 18.80 & 3.18 \\
\hline $2.0 \mathrm{kPa}$ & 27.82 & 0.70 & 34.62 & 4.22 & 48.59 & 1.07 & 5.60 & 2.84 \\
\hline Test factors & $\begin{array}{c}\leq 2 \text { sucked } \\
\text { seed per hill }\end{array}$ & $\begin{array}{c}\text { 1-3 sucked } \\
\text { seed per hill }\end{array}$ & $\begin{array}{c}\geq 6 \text { sucked } \\
\text { seed per hill }\end{array}$ & Average & $\begin{array}{c}\leq 3 \text { sucked } \\
\text { seed per hill }\end{array}$ & $\begin{array}{c}\text { 1-3 sucked } \\
\text { seed per hill }\end{array}$ & $\begin{array}{c}\geq 7 \text { sucked } \\
\text { seed per hill }\end{array}$ & Average \\
\hline $1.2 \mathrm{kPa}$ & 6.70 & 2.67 & 112.74 & 3.21 & 9.23 & 3.30 & 59.58 & 2.51 \\
\hline $1.6 \mathrm{kPa}$ & 22.87 & 1.03 & 22.55 & 2.26 & 11.10 & 1.36 & 9.89 & 2.22 \\
\hline $2.0 \mathrm{kPa}$ & 36.78 & 1.42 & 7.93 & 1.57 & 20.15 & 2.13 & 9.25 & 1.47 \\
\hline
\end{tabular}

\section{Discussion}

The seeder in this study was a seeder with adjustable seeding rates, which can meet the requirements of different seeding rates. Therefore, there were some differences in working parameters under different seeding rates. When 1-3 seeds per hill were used as seeding targets, the optimal sucking negative pressure parameter was $2.0 \mathrm{kPa}$; when 2-4, 3-5, and 4-6 seeds per hill were the seeding targets, the optimal sucking negative pressure parameter was $1.6 \mathrm{kPa}$. The field seeding test is shown in Figure 10.

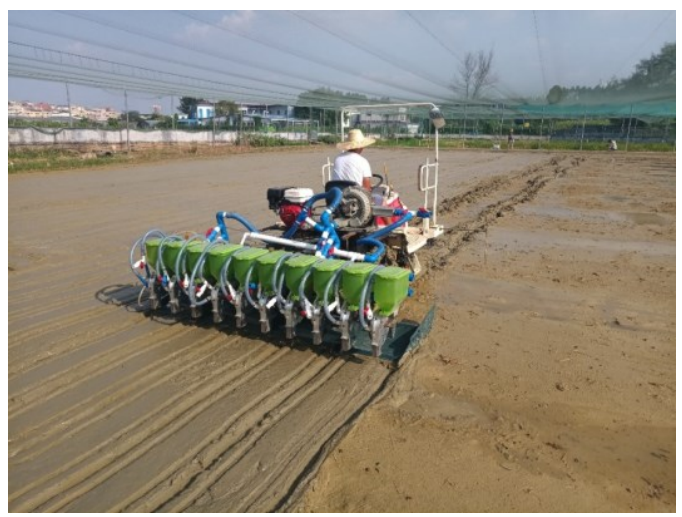

Figure 10 Seeding in the field

The test results revealed that the optimum seed sucking negative pressure was $2.0 \mathrm{kPa}$ when two groups of sucking holes were working. This pressure was higher than the optimum seed sucking negative pressure for 3-5 groups of sucking holes, and this pressure was not consistent with the optimum sucking negative pressure in the laboratory. The main reason for this phenomenon was that there were three negative pressure flow paths in the seed metering device, and each negative pressure flow path worked independently. Because of the mutual movement between the seeding plate and the seed sucking shell, there was a gap between the flow paths and the seeding plate. When two groups of sucking holes were working, only one negative pressure flow path was needed. When 3-5 groups of sucking holes were working, more than two negative pressure flow paths were needed. When one negative pressure flow path was working, the other flow paths did not work and there was no seed sucking negative pressure in those flow paths. The negative pressure of the working flow paths would flow into the non-working flow paths. This would cause negative pressure leakage. When two flow paths worked, only one flow path did not work, and thus the leakage would be reduced. When three flow paths were working, all the flow paths were working, and thus the leakage would be smaller. A seed metering device carried out the test in the laboratory. Because there was only one seed metering device, the negative pressure loss was not obvious, and the optimal negative pressure did not change. However, the rice pneumatic seeder with adjustable seeding rate worked with 10 seed metering devices at the same time, which had negative pressure loss simultaneously, and hence the negative pressure loss would accumulate. When two groups of sucking holes were working, the negative pressure of seed sucking should be increased to ensure the normal operation of the seeder. At the same time, we should further improve the processing technology, improve the airtightness of the seed metering device, and reduce the negative pressure loss.

The results of field tests showed that the seeding precision of each seed metering device was different. The main reason for the difference was the uneven distribution of pipeline pressure, which was consistent with the results of pipeline tests. Because the 
installation position of the metering device on the hill seeder was fixed and unchanged, the seed metering devices in different positions needed pipelines with different lengths. The negative pressure loss changed with the length of the pipeline, which resulted in uneven distribution of air pressure and different seeding effects. Therefore, it was necessary to further optimize the pipeline structure to reduce the loss of pressure. At the same time, the original high-pressure vortex pump could be replaced by several low-pressure vortex pumps. One vortex pump could be used to supply the air source for two seed metering devices, which could effectively reduce the length and bending of the pipeline, and reduce the pressure loss along the way and the local pressure loss. This would lead to a more complex general structure of the seeder.

From the above test results, it could be observed that the seeding effect of the seed metering device has certain fluctuation. The main reason for this fluctuation effect was that the negative pressure of the seeder was measured in the static state in the pipeline pressure test. When the seed metering device worked in the field, the seed metering device was in a dynamic state, and the air pressure inside the seed metering device was unstable and fluctuated greatly. The seeding effect of the seed metering device was also affected by this factor.

\section{Conclusions}

1) In this study, a rice pneumatic seeder with adjustable seeding rates was designed.

2) According to the requirement of negative pressure uniformity, the negative pressure pipeline was optimized by a contrast test.

3) The hybrid rice Wuyou 1179 was selected as the experimental object. Field seeding tests were carried out with different negative pressures and different numbers of sucking holes as experimental factors. The effects of different negative pressures on seeding precision and seeding rate were analyzed. The results showed that the best negative pressure was $2.0 \mathrm{kPa}$ and the average qualified rate was 93.35 when two groups of sucking holes worked, whereas the best negative pressure was $1.6 \mathrm{kPa}$ when three, four, and five groups of sucking holes worked, and the average qualified rate of seeding was $87.21 \%, 83.73 \%$, and $79.86 \%$, respectively. This study provided a research basis for the field application of the adjustable seeding rate of rice.

\section{Acknowledgements}

The authors are thankful for funding from the National Natural Science Foundation of China under Grant No.31871529, the earmarked fund for the National key research program of China under Grant No. 2017YFD07000700, 2017YFD07000704, and China Agriculture Research System under Grant CARS-01-41. The authos also acknowledge the anonymous reviewers for their critical comments and suggestions for improving the manuscript.

\section{[References]}

[1] Farooq M, Siddique K H M, Rehman H, Aziz T, Lee D, Wahid A. Rice direct seeding: Experiences, challenges and opportunities. Soil \& Tillage Research, 2011; 111: 87-98.

[2] Pandey S, Velasco L. Trends in crop establishment methods in Asia and research issues. In: Rice is Life: Scientific Perspectives for the $21 \mathrm{st}$
Century, Proceedings of the World Rice Research Conference, Tsukuba, Japan, 4-7 November 2004; pp.178-181.

[3] Chan C C, Nor M A M. Impacts and implications of direct seeding on irrigation requirement and systems management. In: Paper Presented at the Workshop on Water and Direct Seeding for Rice, 14-16 June 1993. Muda Agricultural Development Authority, Ampang Jajar, Alor Setar, Malaysia.

[4] Yang Z Y, Li N, Ma J, Sun Y J, Xu H. High-yielding traits of heavy panicle varieties under triangle planting geometry: A new plant spatial configuration for hybrid rice in China. Field Crops Research, 2014; 168: 135-147.

[5] Peng S B, Yang J C. Current status of the research on high-yielding and high efficiency in resource use and improving grain quality in rice. Chinese Journal of Rice Science, 2003; 17(3): 275-280.

[6] Luo X W, Jiang E C, Wang Z M, Tang X R, Li J H, Chen W T. Precision rice hill-drop drilling machine. Transactions of the CSAE, 2008; 24(12): 52-56. (in Chinese)

[7] He X, Luo X W, Zang Y, Wang Z M, Cao X M, Liu C B. General structure design and field experiment of pneumatic rice direct-seeder. Int J Agric \& Biol Eng, 2017; 10(6): 31-42.

[8] Zhou Y, Hu M J, Xia J F, Zhang G Z, Xu Z Y, Feng C C, et al. Design and experiment of inside-filling adjustable precision seed-metering device with combined hole for cotton. Transactions of the CSAE, 2018; 34(18): 59-67. (in Chinese)

[9] Zhang M H, Wang Z M, Luo X W, Zang Y, Yang W W, Xing H, et al. Review of precision rice hill-drop drilling technology and machine for paddy. Int J Agric \& Biol Eng, 2018; 11(3): 1-11.

[10] Tian L Q, Wang J W, Tang H, Li S W, Zhou W Q, Shen H G. Design and performance experiment of helix grooved rice seeding device. Transactions of the CSAM, 2016; 47(5): 46-52. (in Chinese)

[11] Ismet O, Degirmencioglu A, Yazgi A. An evaluation of seed spacing accuracy of a vacuum type precision metering unit based on theoretical considerations and experiments. Turkish Journal of Agriculture and Forestry, 2012; 36: 133-144.

[12] Yasir S H. Design and test of a pneumatic precision metering device for wheat. Wuhan: Huazhong Agricultural University, 2011; 134p.

[13] Karayel D. Performance of a modified precision vacuum seeder for no-till sowing of maize and soybean. Soil \& Tillage Research, 2009; 104(2): 121-125.

[14] Karayel D, Barut Z B, Ozmerzi A. Mathematical modelling of vacuum pressure on a precision seeder. Biosystems Engineering, 2004; 87(4): 437-444.

[15] Karayel D, Wiesehoff M, Zmerz A O, Muller J. Laboratory measurement of seed drill seed spacing and velocity of fall of seeds using high-speed camera system. Computers and Electronics in Agriculture, 2006; 50(2): 89-96.

[16] Yazgi A, Degirmencioglu A. Measurement of seed spacing uniformity performance of a precision metering unit as function of the number of holes on vacuum plate. Measurement, 2014; 56(6): 128-135.

[17] Yazgi A, Degirmencioglu A, Onal I, Bayram E. Mathematical modeling and optimization of the performance of a metering unit for precision corn seeding. Journal of Agricultural Machine Science, 2010; 6(2): 107-113.

[18] Zhang G Z, Zang Y, Luo X W, Wang Z M, Zhang Q, Zhang S S. Design and indoor simulated experiment of pneumatic rice seed drilling metering device. Int J Agric \& Biol Eng, 2015; 8(4): 10-18.

[19] Dai Y Z, Luo X W, Zhang M H, Lan F, Zhou Y J, Wang Z M. Design and experiments of the key components for centralized pneumatic rice dry direct seeding machine. Transactions of the CSAE, 2020; 36(10): 1-8. (in Chinese)

[20] Xing H, Zang Y, Wang Z M, Luo X W, Pei J, He S Y, et al. Design and parameter optimization of rice pneumatic seeding metering device with adjustable seeding rate. Transactions of the CSAE, 2019; 35(4): 20-28. (in Chinese)

[21] Cengel Y A, Cimbala J M. Fluid mechanics fundamentals and applications. McGraw-Hill Higher Education, New York, USA, 2013; $1024 \mathrm{p}$.

[22] GB/T 6973-2005. Testing methods of single seed drills (precision drills). National Standards of the People's Republic of China. 2005; 23p. (in Chinese) 